\title{
Biomechanical Analysis of Crossed Pinning Construct on Supracondylar Fracture of Humerus in Children: Does Point of Crossing Matters?
}

Mohd Aizat Azfar Bin Soldin ${ }^{1}$ Mohd Shukrimi bin Awang ${ }^{1}$, Ardilla Hanim Binti Razak ${ }^{1}$ ${ }^{1}$ Department of Orthopaedics, Traumatology and Rehabilitation, Kulliyyah of Medicine, International Islamic University Malaysia

Presenter: Mohd Aizat Azfar Soldin

Introduction: Percutaneous pin fixation either by crossed pinning construct (CPC) or lateral divergent pinning construct (LDPC) are the recommended treatment for displaced supracondylar humerus fractures (SCHF) in children. Several studies had compared the biomechanical stability between these two, however, a biomechanical analysis of varying crossing point location in CPC has not been performed previously. The aim of this study was to compare the stability of various crossing point location in CPC and LDPC. Materials and Methods: Thirty synthetic humeri were osteotomized at mid olecranon fossa, anatomically reduced and pinned using two $1.6 \mathrm{~mm}$ Kirschner wiresin five different constructs namely centre point, medial point, lateral point, superior point and LDPC. Six samples were prepared for each construct and were tested for extension, flexion, valgus, varus, internal rotation and external rotation forces by using Universal Tensile Machine and the data were analysed with R Statistic. Results: The centre point CPC was the stiffest while the LDPC and medial point CPC were the least stiff construct for linear and rotational force respectively. Lateral point CPC, and superior point CPC showed no statistically significant difference when compared to centre point CPC. Conclusion: The centre point CPC was the most stable construct while the LDPC and medial point CPC were the least stable. The stability of lateral point CPC and superior point CPC were statistically comparable to centre point CPC. Clinically, this will help the treating surgeon to reduce the numbers of attempt during $\mathrm{K}$-wire insertion in order to get perfect centre point CPC. 